\title{
Una nueva solución al problema de la fundamentación
}

\author{
A new solution to the grounding problem
}

\author{
Marta Campdelacreu \\ Departament de Filosofia, Universitat de Barcelona, España \\ marta_campdelacreu@ub.edu
}

\begin{abstract}
Resumen
Consideremos una estatua y el trozo de arcilla de que está hecha. Supongamos que empiezan a existir y dejan de existir en el mismo momento de tiempo. Según el co-ubicacionismo la estatua y el trozo de arcilla son dos objetos diferentes: tienen propiedades diferentes (por ejemplo, uno es una estatua y el otro un trozo de arcilla) y según la Ley de Leibniz, un mismo objeto no puede tener propiedades diferentes. Una de las preguntas más difíciles de responder para el co-ubicacionismo es la del problema de la fundamentación. Dado que la estatua y el trozo de arcilla comparten muchas de sus propiedades (su materia, su composición microscópica, su estructura, etc.), ¿qué fundamenta el hecho de que tengan diferentes propiedades clasificatorias (o modales)? Recientemente, Catherine Sutton ha ofrecido una respuesta muy interesante a la pregunta. Sin embargo, como argumentaremos, no puede aplicarse a todos los casos de objetos co-ubicados y, por lo tanto, no es una solución adecuada al problema de la fundamentación. El objetivo principal de este artículo es presentar una nueva solución al problema de la fundamentación que integre alguna de las tesis de Sutton, pero que nos permita dar una respuesta completa a la pregunta. Para ello será crucial la noción de proceso para empezar a existir. Una vez presentada la nueva propuesta la compararemos con las de Kit Fine y Noël Saenz.
\end{abstract}

Palabras clave: co-ubicacionismo, propiedad clasificatoria, proceso para empezar a existir, Catherine Sutton, Kit Fine, Noël Saenz.

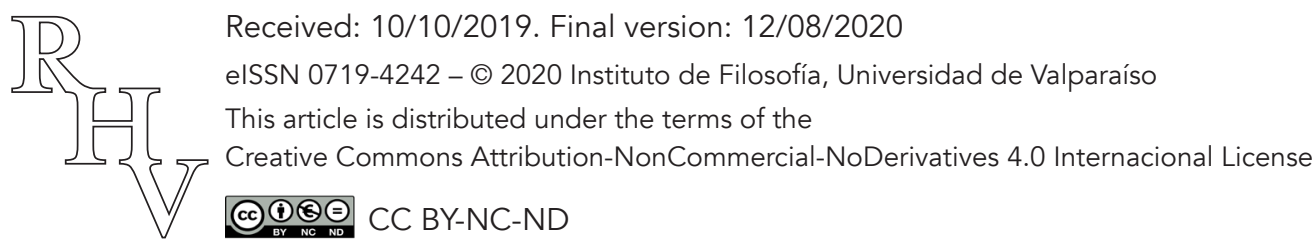




\begin{abstract}
Let us consider a statue and the piece of clay out of which it is made, and let us suppose that they start to exist and cease to exist at exactly the same time. According to colocationism, the statue and the piece of clay are two different objects: they have different properties (for example, one is a statue and the other a piece of clay) and, according to Leibniz's Law, the same object cannot have different properties. One of the most difficult questions for colocationism is that of the grounding problem: given that the statue and the piece of clay share many of their properties (their matter, their microscopic composition, their structure, etc.), what is it that grounds the fact that they have different sortal (or modal) properties? Recently, Catherine Sutton has offered a very interesting answer to the question. However, as I will argue, it cannot be applied to all cases of colocated objects and therefore, it is not an adequate solution to the grounding problem. The main objective of this paper is to present a new solution to the grounding problem that integrates some of Sutton's theses, but that allows us to give a complete answer to the question. To do this, the notion of a process of coming into existence will be crucial. After presenting the new proposal, I will compare it with the proposals by Kit Fine and Noël Saenz.
\end{abstract}

Keywords: colocationism, sortal property, process of coming into existence, Catherine Sutton, Kit Fine, Noël Saenz.

\title{
1. Introducción
}

El sentido común parece indicarnos que hay estatuas, sillas, árboles, gatos, personas, trozos de diferentes materiales, etc. Entonces, parece que resulta perfectamente pertinente preguntarnos, por ejemplo: ¿cuál es la relación entre una estatua y el trozo de arcilla del que está hecha, o entre una silla y el trozo de madera del que está hecha?

Una razón poderosa que tradicionalmente se ha ofrecido a favor de la tesis de que, por ejemplo, la estatua y el trozo de arcilla son dos objetos numéricamente distintos es la siguiente (cf. Gibbard 1975): imaginemos una estatua, ESTATUA, creada y destruida al mismo tiempo que el trozo de arcilla, ARCILLA, del que está hecha. ESTATUA y ARCILLA tienen propiedades diferentes -por ejemplo, sus propiedades clasificatorias (así es como proponemos traducir 'sortal properties') o modales: uno es un trozo de arcilla y el otro una estatua y, así, uno puede cambiar completamente su forma pero el otro no (cf. Fine 2003) - y si tienen propiedades diferentes, según la Ley de Leibniz, son objetos diferentes. (cf. Wasserman 2017, Campdelacreu 2015).

El co-ubicacionismo (así proponemos traducir el vocablo inglés 'colocationism') acepta que, en términos de nuestro ejemplo, ESTATUA y ARCILLA son dos objetos diferentes y, en general, afirma que dos o más objetos materiales diferentes pueden estar compuestos, al mismo tiempo, de las mismas partes. Esto sería así, al menos, para cierto 
nivel de descomposición de los objetos en partes. Así, los objetos co-ubicados también compartirían, en ese instante, la materia de la que están hechos y la región donde están ubicados. (Otras propuestas como el nihilismo u otras basadas en la teoría de las contrapartidas no aceptan que ESTATUA y ARCILLA sean numéricamente distintos y, para ello, por ejemplo, propuestas del primer tipo niegan que, de hecho, existan tales objetos, o, en el caso de las propuestas del segundo tipo, proponen entender a las propiedades modales como siendo relativas a propiedades clasificatorias. En este artículo nos centraremos, no obstante, solo en el análisis y discusión del co-ubicacionismo. cf. Wasserman 2017, Campdelacreu 2015)).

Una de las preguntas más difíciles de responder para el co-ubicacionismo es la correspondiente al problema de la fundamentación (aquí nos centraremos en este problema pero otros problemas para la posición serían los relacionados con la intuición de que no puede haber dos objetos diferentes en la misma región espaciotemporal o con la intuición de que no puede haber dos objetos diferentes con las mismas partes propias). Sutton (2012) formula el problema de la fundamentación de la siguiente manera:

¿qué fundamenta la diferencia en propiedades (por ejemplo, en propiedades clasificatorias o modales) de ESTATUA y ARCILLA? Pues, Sutton dice, estos objetos 'se encuentran en el mismo entorno y adquieren propiedades a partir de las mismas partes componentes. Pero las diferencias en propiedades deberían estar fundamentadas.' (Sutton 2012, 703-704, traducción nuestra).

Sutton ofrece una respuesta al problema que, argumentaremos, es, en lo esencial, correcta para cierto tipo de casos, pero que no puede aplicarse a otro tipo de casos que, como argumento en (Campdelacreu 2016), son tan reales como los primeros. Así, su solución es incompleta, y por tanto, incapaz de solventar el problema. El objetivo principal de este artículo es presentar una nueva solución al problema de la fundamentación que incluya aquellas tesis de Sutton que, veremos, son esencialmente correctas, y que las complemente adecuadamente, de forma que dispongamos de una propuesta que pueda aplicarse a todos los tipos de casos de objetos materiales co-ubicados. ${ }^{2}$ ).

El contenido del artículo es el siguiente: en la Sección 2, presentaremos la solución de Sutton al problema de la fundamentación y veremos resumidamente lo que defiendo en (Campdelacreu 2016) para mostrar que su solución es incompleta y, que, por lo tanto, es insatisfactoria. En la Sección 3, elaboraremos una nueva propuesta que preservará

\footnotetext{
${ }^{1}$ Para otras formulaciones y propuestas de solución véase el artículo de Sutton. Como explicaremos en el texto principal, en este artículo vamos a centrarnos básicamente en la de Sutton aunque la compararemos brevemente con las de Fine y Saenz.

${ }^{2}$ Dejamos como trabajo para el futuro la presentación de una comparación detallada de la propuesta que aquí presentamos con el resto de propuestas en la literatura. Esta tarea es necesaria, claro, para una defensa completa de la propuesta que esbozamos aquí.
} 
algunas de las tesis de Sutton pero que, al contrario de lo que pasa con su propuesta, argumentaremos, nos proporciona una respuesta completa, y así satisfactoria, al problema de la fundamentación. Finalmente, en la Sección 4, formularemos y responderemos brevemente a algunas posibles objeciones a la nueva propuesta y la compararemos con las de Kit Fine y Noël Saenz.

\section{La respuesta de Sutton al problema de la fundamentación}

A continuación vamos a exponer la solución de Sutton, en (Sutton 2012), al problema de la fundamentación. Antes de empezar, no obstante, debemos apuntar un par de cosas. Primero, Sutton usa el concepto de fundamentación (grounding en inglés) de una manera diferente a la que vamos a proponer. Sin embargo, en esta sección, vamos a seguir su uso. Más adelante introduciremos la manera cómo vamos a entender el concepto y explicaremos las razones. Segundo, de ahora en adelante supondremos que los casos de objetos co-ubicados que mencionemos son casos de objetos que se hallan co-ubicados a lo largo de toda su existencia. Que haya una solución al problema de la fundamentación depende de que exista también para estos casos, donde no podemos apelar a diferentes propiedades temporales de los objetos para solucionarlo.

Sutton empieza presentando dos definiciones:

Composición extrínseca: un objeto $O$ está extrínsecamente compuesto si y solo si la composición de $O$ (y así, la existencia de $O$ ) está fundamentada en parte en relaciones que las partes de $O$ mantienen con entidades que no son parte de $O$.

Composición intrínseca: un objeto $O$ está intrínsecamente compuesto si y solo si la composición de $O$ (y así, la existencia de $O$ ) no está fundamentada, ni siquiera en parte, en relaciones que las partes de $O$ mantienen con entidades que no son partes de $O$.

Nótese que en estas definiciones Sutton omite cualquier referencia a momentos de tiempo. Una pregunta interesante en relación a esta definición propuesta por Sutton es, pues, la siguiente. ¿En qué momentos de tiempo las partes de un objeto extrínsecamente compuesto deben mantener las relaciones adecuadas con las entidades que no son parte del mismo? ¿Solo cuando el objeto empieza a existir o más bien en todo momento de tiempo? Sutton no considera esta cuestión en su artículo (2012, 710 y ss). Por lo que dice parece claro que las partes del objeto deben mantener tales relaciones en el momento en que el objeto compuesto empieza a existir. Pero no queda claro qué debe pasar en el resto de momentos en los cuales el objeto existe. Aunque es un tema muy interesante, en este artículo deberemos dejar la discusión meramente anotada, pues nos desviaría del tema principal, y la solución que aporta Sutton al problema de la fundamentación no parece depender de cómo finalmente se decida resolver la cuestión. 
Volviendo, pues, a la solución de Sutton al problema de la fundamentación, Sutton mantiene que en todos los casos de co-ubicación, al menos uno de los objetos está extrínsecamente compuesto. Es más, cuando los dos están extrínsecamente compuestos, las relaciones que sus partes compartidas mantienen con los objetos externos son diferentes. Así, su respuesta al problema de la fundamentación distingue los siguientes dos tipos de casos.

Primero, aquellos casos en que uno de los dos objetos está extrínsecamente compuesto y el otro intrínsecamente compuesto. Este es el caso, por ejemplo, de los trozos de materia, que Sutton considera como extrínsecamente compuestos (dado que su existencia está fundamentada en las relaciones que sus partes propias mantienen con ciertos estados mentales sobre trozos de materia) co-ubicados con objetos naturales inanimados, que Sutton considera que están intrínsecamente compuestos (como por ejemplo, Sutton afirma, los diamantes, los planetas, etc.). En este tipo de casos, afirma Sutton, la diferencia en las propiedades clasificatorias de los objetos está fundamentada en las diferentes relaciones en que están las partes compartidas: las partes del objeto extrínsecamente compuesto mantienen relaciones con objetos externos, las partes del objeto intrínsecamente compuesto no.

Segundo, aquellos casos en que los dos objetos están extrínsecamente compuestos. Este es el caso, por ejemplo, de los trozos de materia co-ubicados con, por ejemplo, una estatua (como ARCILLA y ESTATUA), o con otros artefactos (que Sutton considera que están extrínsecamente compuestos, dado que su existencia está fundamentada en las relaciones que sus partes mantienen con ciertos estados mentales sobre el tipo relevante de artefacto). Este es el caso también de los animales y los trozos de materia adecuada con los cuales están co-ubicados (los animales están extrínsecamente compuestos porque su existencia está fundamentada, al menos en parte, en las relaciones extrínsecas que sus partes mantienen con otros miembros de la especie en cuestión). En este tipo de caso los dos objetos co-ubicados comparten sus partes pero las relaciones que estas mantienen con objetos externos son diferentes para cada objeto. Estas diferentes relaciones fundamentan la diferencia en sus propiedades clasificatorias.

Esta es, pues, la solución de Sutton al problema de la fundamentación. Obviamente, no es válida para los casos en que los dos objetos co-ubicados están intrínsecamente compuestos. En (Campdelacreu 2016) argumento que, en contra de lo que afirma Sutton, este tipo de casos sí son posibles. Así, la solución de Sutton sería incompleta, y por lo tanto (al menos parcialmente) inadecuada. A continuación voy a exponer, de manera resumida, útil para nuestros propósitos actuales, lo que defiendo allí.

La primera cosa relevante que debemos notar es que Sutton no dice nada sobre cierto tipo de casos que, aunque parece plausible negar que existan en el mundo real, no es claro que no sean, sin embargo, posibles. Por ejemplo, Sutton acepta que los objetos naturales inanimados en general están intrínsecamente compuestos. Sus ejemplos son los diaman- 
tes y los planetas. Asumo que aceptaría como siendo del mismo tipo a las montañas o las fallas, y a las esmeraldas y los rubíes. Por ejemplo, Sutton dice sobre un diamante que toma como ejemplo:

El diamante parece un buen candidato a composición intrínseca. La existencia de diamantes no depende de las intenciones humanas sobre los diamantes. Los diamantes fueron descubiertos, no inventados. Mi solución al problema de la fundamentación no discute la presunta composición intrínseca de los tipos naturales inanimados. (Sutton 2012, 719, traducción nuestra)

Pero entonces, aunque en el mundo real no haya montañas co-ubicadas con rubíes o fallas co-ubicadas con diamantes, esto no significa que su existencia no sea metafísicamente posible. Sutton no dice nada sobre estos casos. Casos que involucran objetos co-ubicados intrínsecamente compuestos. Para ellos, como ya hemos visto, la solución de Sutton no es válida. Creo que esto, en sí mismo, ya es una razón para creer que la propuesta de Sutton tiene que ser enmendada.

De hecho, argumento en el artículo citado, también existen casos de objetos co-ubicados intrínsecamente compuestos que se dan en el mundo real. Para mostrarlo argumento primero, en contra de Sutton, que los trozos de materia no están extrínsecamente compuestos. Como hemos visto más arriba, Sutton afirma que estos están extrínsecamente compuestos porque su composición (y existencia) está fundamentada, al menos en parte, en las relaciones que sus partes mantienen con ciertos estados mentales sobre trozos de materia.

Veamos, resumidamente, las razones que ofrece Sutton para la tesis de que los trozos de materia están extrínsecamente compuestos y las razones que, creo, nos deben llevar, en última instancia, a rechazarla. Creo que, de acuerdo con el sentido común, podría existir un mundo sin humanos pero repleto de trozos de materia. Nuestras intuiciones parecen favorecer claramente la tesis que se trata de objetos intrínsecamente compuestos y, normalmente, los filósofos han mantenido esta posición. Sutton lo reconoce y ofrece un argumento para mostrar que la tesis es, sin embargo, incorrecta. Su argumento, básicamente, es el siguiente: asumamos que los trozos de materia están intrínsecamente compuestos. Así, probablemente, sus condiciones para empezar a existir consisten en que sus partes estén en contacto y se muevan como una unidad. Pero entonces, cuando pegamos una pegatina a una fiambrera, debería empezar a existir un nuevo objeto teniéndoles como partes propias; sin embargo, añade Sutton, cuando esto pasa, no reconocemos la existencia de ningún nuevo objeto. La propuesta de Sutton es que se necesita alguna cosa más para que un trozo de materia empiece a existir: que sus partes mantengan una relación extrínseca adecuada con ciertos estados mentales sobre los trozos de materia.

Creo que el argumento de Sutton no es convincente por las siguientes razones. Su pregunta es sobre si, en una situación como la descrita, nuestras intuiciones son que un 
nuevo objeto empieza a existir y tiene a la fiambrera y a la pegatina como partes propias. Sin embargo, creo que, para evaluar la tesis que los trozos de materia están intrínsecamente compuestos, la pregunta debería ser ligeramente, pero relevantemente, diferente. Esto es así por dos razones: primero, lo que estamos discutiendo son las condiciones de existencia de un tipo de objeto muy específico, un trozo (o pedazo) de materia. Se trata de objetos a los cuales raramente prestamos atención en nuestra vida cotidiana. Así, cuando la pregunta se formula, se tiene que enfatizar que el contexto es uno en el que este tipo de objeto es también relevante. Sutton no lo hace. Segundo, la pregunta de Sutton es sobre el empezar a existir de un objeto que tiene como partes propias la fiambrera y la pegatina. Pero creo que el empezar a existir de un trozo de materia en una situación como esta no requiere que las partes propias sean la fiambrera y la pegatina; podrían ser, por el contrario, solamente los trozos de materia de los que estas están hechas, pues la idea que las partes propias de los trozos de materia son solo trozos de materia más pequeños parece ser perfectamente compatible con una teoría co-ubicacionista. Sin embargo, Sutton parece excluir esta posibilidad. En resumen, creo que la pregunta de Sutton, para ser válida para evaluar la tesis que los trozos (pedazos) de materia están intrínsecamente compuestos, debería ser formulada en los siguientes términos (o equivalentes): supongamos que estamos en un contexto en el cual (por las razones que sean) los trozos de materia también son relevantes. Supongamos que pegamos la pegatina a la fiambrera. ¿Según nuestras intuiciones, existe un nuevo trozo de materia compuesto de, al menos, los trozos de materia más pequeños de los cuales la fiambrera y la pegatina están hechas? Decimos 'al menos' porque también puede haber teorías co-ubicacionistas según las cuales la fiambrera y la pegatina también son partes propias del recién creado trozo de materia. Ahora, si la pregunta se formula en estos términos y contexto, creo que nuestras intuiciones ya no son las que dice Sutton sino más bien que sí existe un nuevo trozo de materia.

Ahora bien, si esto es así, es fácil imaginar casos en el mundo real de objetos co-ubicados intrínsecamente compuestos: por ejemplo, el de un iceberg co-ubicado con un gran trozo de hielo, o como veremos más adelante, el de un diamante co-ubicado con un trozo de materia del tipo adecuado. Recordemos que, como hemos visto antes, Sutton afirma que, en general, los objetos naturales inanimados están intrínsecamente compuestos y estos objetos están co-ubicados, en general, con trozos de materia del tipo adecuado.

En conclusión, en contra de lo que afirma Sutton, parece haber buenas razones para afirmar que existen casos de objetos co-ubicados intrínsecamente compuestos. Así, su respuesta al problema de la fundamentación no es (completamente) adecuada.

\section{Una propuesta (parcialmente) nueva}

En esta sección vamos a formular una nueva propuesta, integrando alguna de las tesis de Sutton, que tenga también en cuenta los casos en que los dos objetos co-ubicados están intrínsecamente compuestos. Primero analizaremos los elementos que la nueva pro- 
puesta comparte con la de Sutton y presentaremos la manera exacta en que proponemos integrarlos en la propia. Después desarrollaremos la nueva propuesta, añadiendo nuevas tesis, para poder responder al problema de la fundamentación para los casos de objetos co-ubicados intrínsecamente compuestos.

\subsection{ESTATUA y ARCILLA}

Vamos a presentar las tesis que, básicamente, compartimos con Sutton comentando el siguiente texto en el que describe un caso como el de ESTATUA y ARCILLA -asumiremos que la estatua de la que habla es ESTATUA y el trozo de arcilla es ARCILLA, pues esto simplificará la discusión.

[C]iertos objetos, los $x \mathrm{~s}$, mantienen una relación extrínseca con las intenciones humanas sobre estatuas, y en virtud de esa relación, se compone y empieza a existir una estatua. Estos mismos $x$ s mantienen una relación extrínseca diferente con las intenciones humanas sobre trozos [de arcilla], y en virtud de esa relación, el trozo [de arcilla] se compone y empieza a existir. (Sutton 2012, 175, traducción nuestra)

La nueva propuesta que vamos a presentar comparte con la de Sutton la relevancia de los que ella llama 'los $x \mathrm{~s}$ ', las futuras partes compartidas de los objetos co-ubicados y también que estos estén relacionados con los estados mentales relevantes sobre las estatuas. Como ya he explicado y justificado, creo que para los trozos (pedazos) de materia no existen las relaciones que Sutton postula.

Antes de explicar cómo vamos a integrar exactamente en nuestra propuesta las tesis de Sutton, necesitamos formular la siguiente precisión terminológica. En el artículo citado previamente, Sutton no explicita como entiende exactamente los relata de la relación de fundamentación y, en todo caso, no los restringe a hechos, como nosotros haremos aquí siguiendo a muchos otros filósofos en esto (cf. Correia y Schnieder 2012; Trogdon 2013; Bliss y Trogdon 2014; Raven 2015).

Una consecuencia de esta discrepancia es como se formula exactamente el problema de la fundamentación -cabe decir, pero, que lo que sigue puede ser adaptado a diferentes propuestas sobre cuales sean los relata de la relación de fundamentación, como nosotros haremos ahora con algunas tesis de la propuesta de Sutton (véanse, por ejemplo, deRosset 2013 y Saenz 2015, en relación a la existencia de conexiones sistemáticas entre maneras diferentes de entender los relata de la relación). De acuerdo con lo dicho sobre la relación de fundamentación, la formulación sería la siguiente: ¿qué hecho fundamenta el hecho de que (por ejemplo) ESTATUA y ARCILLA tengan propiedades clasificatorias diferentes? Porque este hecho no parece un hecho que no requiera ser fundamentado, básico. Sin 
embargo, también parece ser un hecho que ESTATUA y ARCILLA están ubicados en el mismo entorno y que poseen propiedades en virtud de tener ciertas partes componentes, que son las mismas.

Debemos señalar también que las afirmaciones que hagamos involucrando la relación de fundamentación entre hechos pretenden ser compatibles con diferentes propuestas sobre la naturaleza exacta de esta relación. Con todo, asumiremos sin más argumentación, pues esto nos apartaría demasiado del tema principal de este artículo, que la noción de fundamentación está estrechamente ligada a la noción de explicación (metafísica); por ejemplo, en este artículo nos interesa qué explica que, por ejemplo, ESTATUA y ARCILLA tengan propiedades clasificatorias diferentes. Una vez presentada la propuesta señalaremos otra presuposición importante que, aun así, por los mismos motivos, aquí deberá quedar meramente introducida.

Ahora, ¿cómo proponemos integrar en la nueva propuesta aquellas tesis de Sutton que, esencialmente, compartimos? La propuesta es hacerlo en términos de lo que vamos a llamar 'procesos para empezar a existir', que vamos a abreviar, por comodidad, como 'pro$\operatorname{cesos}^{\mathrm{e}}$ ' (la 'e' de empezar a existir). Primero vamos a presentarlos en términos generales y luego para el caso de ESTATUA y ARCILLA. A continuación veremos como las mismas tesis generales pueden aplicarse a todos los otros tipos de casos de co-ubicación. Una vez hayamos presentado la propuesta para todos los casos, formularemos y responderemos a posibles objeciones (sección 4).

La propuesta es que los objetos materiales en cuestión aquí son el resultado de ciertos procesos adecuados. El proceso para empezar a existir de las estatuas es diferente al proceso $^{\text {e }}$ de los olmos o de los loros o de los seres humanos o de los diamantes o de los trozos de arcilla, etc.

En general, los procesose consumen tiempo. Ahora, en este artículo me centraré en la última parte de estos procesos, que serán las relevantes para nuestros propósitos. Tener en cuenta todas sus partes haría la presentación de la propuesta más difícil de entender, pero no añadiría ningún elemento relevante nuevo. Así, de ahora en adelante, asumiré que estamos hablando solo de la última parte de tales procesos.

A continuación vamos a presentar los tipos de componentes de estos procesos ${ }^{\mathrm{e}}$ que son cruciales para la respuesta al problema de la fundamentación. Puede haber otros tipos de componentes pero nos centraremos exclusivamente en los relevantes para nuestros propósitos.

Un proceso ${ }^{\mathrm{e}}$ de un objeto material tiene entre sus componentes las partes materiales futuras del objeto, cuando este empiece a existir (y que pueden cambiar, todas o algunas, posteriormente). Las llamaremos, entendidas en su conjunto, 'el componente material' de un proceso $^{\mathrm{e}}$. Todos los procesos ${ }^{\mathrm{e}}$ de objetos materiales tienen entre sus componentes su respectivo componente material. Una característica especial de los componentes ma- 
teriales es que diferentes procesos ${ }^{\mathrm{e}}$ de diferentes objetos pueden compartirlos. Este es el caso, por ejemplo, de ESTATUA y ARCILLA. De esta manera precisa que acabamos de ver propongo integrar en la nueva propuesta la afirmación de Sutton de que los xs que mantienen las relaciones apropiadas con las intenciones humanas sobre estatuas son exactamente los mismos xs que están en las relaciones apropiadas con (en la propuesta de Sutton) otras intenciones humanas sobre los trozos de arcilla. En mi propuesta las relaciones involucradas en el proceso ${ }^{e}$ del trozo de arcilla no serán con intenciones humanas sino, de hecho, relaciones que los xs mantienen entre sí.

Otros dos tipos de componentes de los procesos ${ }^{\mathrm{e}}$ que serán importantes para nuestros propósitos son las relaciones externas que el componente material puede mantener con entidades externas a él, y las relaciones internas que los componentes del componente material mantienen entre sí.

Presentaré primero las relaciones externas que un componente material puede mantener con otras entidades que no son parte de él. Este tipo de componente está presente en los procesos ${ }^{\mathrm{e}}$ cuyos resultados son objetos como estatuas, monumentos, tijeras, etc., y a lo mejor, personas, caballos, árboles, y otros seres animados (véase el artículo de Sutton para la tesis que los objetos animados están extrínsecamente compuestos.), etc. Por ejemplo, en el caso de ESTATUA, el componente material del proceso $^{\mathrm{e}}$ del cual es resultado estuvo relacionado de forma adecuada con el artista, sus intenciones y acciones, etc. (qué relaciones exactamente son estas es algo que la teoría del arte correcta, la parte relevante para las estatuas, dilucidará). Como vimos antes, creo (contra Sutton) que este no es un

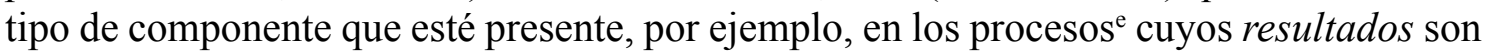
los trozos de materia.

De esta manera que acabamos de ver es como propongo integrar en la nueva propuesta las tesis de Sutton sobre las estatuas que hemos presentado antes.

Como hemos visto arriba, en el mismo texto, Sutton defiende que los mismos $x s$ mantienen relaciones extrínsecas con intenciones humanas sobre trozos de arcilla y que, en virtud de esto, un trozo de arcilla, ARCILLA, empieza a existir. Creo que es en este punto donde Sutton se equivoca, como hemos argumentado antes. Nuestra propuesta para el caso de ARCILLA, y otros objetos como este, es la siguiente (en la sección siguiente la refinaremos).

Entre los componentes de los procesos ${ }^{\mathrm{e}}$ figuran las relaciones internas que las partes propias del componente material mantienen entre sí. Estas relaciones internas pueden ser diferentes para diferentes tipos de procesos $^{\mathrm{e}}$. Por ejemplo, para los procesos ${ }^{\mathrm{e}} \operatorname{cuyos}$ resultados son trozos de mármol y para aquellos cuyos resultados son trozos de materia de átomos de carbono. Los expertos determinaran el tipo exacto de relación presente en cada tipo de proceso $^{\mathrm{e}}$. Así, por ejemplo, ARCILLA es el resultado de un proceso que 
tiene como componentes a los $x s$ y a las relaciones internas adecuadas que estos mantienen entre sí (los expertos en procesos ${ }^{\mathrm{e}}$ de trozos de arcilla determinaran cuales son estas relaciones internas).

Ahora, veamos como lo que hemos introducido hasta aquí nos permite formular una respuesta al problema de la fundamentación para el caso de ESTATUA y ARCILLA. De esta manera quedará también patente la segunda presuposición sobre la relación de fundamentación, tal y como habíamos apuntando más arriba.

El primer punto relevante es el siguiente. Por ejemplo, para ESTATUA, diremos que el hecho que fundamenta el hecho de que ESTATUA tenga la propiedad clasificatoria de ser una estatua es el hecho de que el proceso $^{e}$ del cual es resultado tenga los componentes adecuados, en particular, que su componente material mantenga las relaciones con las intenciones y acciones del artista adecuadas, etc.

Queda patente, pues, la última presuposición sobre la relación de fundamentación a la que aludíamos más arriba: en contra de lo que ha sido más frecuente suponer en la literatura sobre esta relación, propongo entender a esta relación de fundamentación entre hechos como siendo, para los casos que estamos tratando aquí, una relación diacrónica, en el sentido que los hechos fundamentadores y los hechos fundamentados no refieren al mismo momento de tiempo. Es verdad que para muchos ámbitos la relación parece ser claramente sincrónica (por ejemplo, cuando se afirma que el hecho de que algo sea rojo oscuro fundamenta el hecho de que sea rojo) pero no veo ninguna razón para pensar que esto deba ser así para todos. Aunque aquí no podremos más que dejar apuntada esta presuposición, puede consultarse (cf. Bliss y Trogdon 2014) para ejemplos (que proporcionan al principio del artículo) que se ofrecen como siendo de fundamentación que involucran hechos que presentan esta misma característica (cf. Trogdon 2018, 1034-1035; Wilson 2018) y para breves discusiones (en un contexto diferente al nuestro) poniendo en duda la idea hasta ahora más estándar y en favor de la posibilidad de una relación de fundamentación que no necesariamente tenga que ser, para todos los ámbitos de aplicación, una relación de carácter sincrónico. Una cuestión interesante aquí es si la relación de fundamentación en su versión diacrónica presenta las mismas propiedades modales que la relación de fundamentación en su versión sincrónica. Aunque una justificación detallada nos llevaría demasiado lejos de nuestro objetivo principal en este artículo, aquí quisiera dejar apuntado que, como se defiende mayoritariamente para el caso de la relación en su versión sincrónica, la relación de fundamentación en su versión diacrónica tal y como la entendemos aquí cumple que: si los hechos fundamentadores se dan, luego, necesariamente, los hechos fundamentados se dan.

Ahora, para el caso de ARCILLA, diremos que el hecho que fundamenta el hecho de que ARCILLA tenga la propiedad clasificatoria de ser un trozo de arcilla es el hecho de que el proceso ${ }^{e}$ del cual es resultado tiene un componente material (que comparte con el proceso ${ }^{\mathrm{e}}$ cuyo resultado es ESTATUA) cuyos componentes mantienen las relaciones 
internas del tipo adecuado (que determinarán los expertos). Por otro lado, no es un componente del proceso ${ }^{\mathrm{e}}$ cuyo resultado es ARCILLA las relaciones extrínsecas que sí son un componente del proceso ${ }^{\mathrm{e}}$ cuyo resultado es ESTATUA.

En definitiva, pues, el hecho de que ESTATUA y ARCILLA tengan diferentes propiedades clasificatorias está fundamentado en el hecho de que los procesos ${ }^{\mathrm{e}}$ de los cuales ESTATUA y ARCILLA son resultados difieren en sus componentes. Como acabamos de ver, el hecho de que un proceso ${ }^{\mathrm{e}}$ tenga ciertos componentes y no otros fundamenta el hecho de que el objeto resultante tenga cierta propiedad clasificatoria y no otra.

Una cuestión importante que puede surgir en este punto es la siguiente: ¿es realmente una respuesta aceptable al problema de la fundamentación una propuesta que apele en cierto momento a hechos metafísicamente básicos, en el sentido de primitivos? Por ejemplo, dos parágrafos más arriba hemos considerado como tal al hecho de que las relaciones

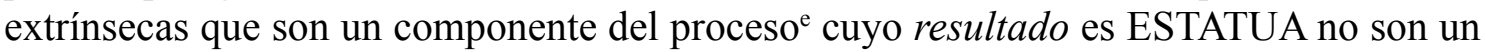
componente del proceso ${ }^{\text {e }}$ cuyo resultado es ARCILLA. En nuestra opinión, como pasa en muchos, quizás todos, los casos de explicación, en algún estadio u otro de la misma se deberá aceptar que se ha llegado a algo metafísicamente básico, primitivo. Otra cosa es comparar y argumentar a favor de alguna de las diferentes propuestas sobre qué es metafísicamente básico, primitivo.

Antes de continuar con la presentación de los nuevos elementos de la propuesta, me gustaría detenerme un instante en un par de cuestiones.

Primero, la cuestión de qué tipo de entidades son estos procesos. De hecho lo que me gustaría decir es que aunque hemos asumido su existencia, que vamos a defender más adelante, y hemos dado algunos detalles sobre sus componentes, en este artículo querría mantenerme tan neutral como fuera posible sobre qué tipo exacto de entidad son. Nuestra intención aquí es que las afirmaciones sobre ellos sean compatibles con diferentes teorías sobre su naturaleza. En exactamente la misma línea, hemos dicho que un objeto material es el resultado de un proceso ${ }^{\text {e }}$. Pero, ¿en qué consiste exactamente esta relación de ser el resultado de? Como antes, aquí asumiremos que existe esta conexión entre procesos ${ }^{\mathrm{e}} \mathrm{y}$ objetos pero, dado el objetivo del artículo, permaneceremos neutrales sobre la naturaleza exacta de esta.

Segundo, relacionado con lo que acabamos de decir, quisiera enfatizar otra vez que la propuesta no es que las propiedades clasificatorias de los objetos son el resultado de estos procesos $^{\text {e }}$. El resultado de estos procesos son los objetos. Y la propuesta es que el hecho de que un proceso ${ }^{\mathrm{e}}$ tenga ciertos componentes y no otros fundamenta el hecho de que el objeto resultante tenga cierta propiedad clasificatoria y no otra. 
Ahora, como ya hemos explicado antes, la propuesta de Sutton no es adecuada para explicar por qué objetos co-ubicados, ambos intrínsecamente compuestos, pueden tener propiedades clasificatorias diferentes. En el resto de esta sección mostraremos cómo la propuesta que hemos empezado a presentar puede aplicarse también a este tipo de caso.

Antes debemos apuntar que, dado como hemos propuesto integrar las ideas de Sutton en nuestra propuesta, vamos a entender a partir de ahora la distinción entre objetos intrínsecamente/ extrínsecamente compuestos de la siguiente manera:

Composición extrínseca: un objeto $O$ está extrínsecamente compuesto si y solo si esel resultado de un proceso ${ }^{e}$ que tiene como componente relaciones con entidades que no son componentes del componente material del proceso ${ }^{e}$.

Composición intrínseca: un objeto $O$ está intrínsecamente compuesto si y solo si esel resultado de un proceso $^{e}$ que no tiene entre sus componentes relaciones con entidades que no son componentes del componente material del proceso ${ }^{e}$.

\subsection{DIAMANTE y TROZO-C}

Para formular la respuesta al problema de la fundamentación para este tipo de casos, vamos a usar un ejemplo de Sutton (ligeramente modificado) del diamante al que ya hemos aludido más arriba al presentar la propuesta de Sutton -de esta manera podemos seguir comparando las dos propuestas.

Sutton presenta su solución al problema de la fundamentación en tres partes, según el tipo de objetos involucrados. En primer lugar analiza casos como el ya visto de ESTATUA y ARCILLA en los cuales están involucrados dos artefactos. En segundo lugar analiza casos en los que están involucrados objetos naturales inanimados, como es el caso del diamante, co-ubicados con masas (cf. Sutton 2012, 719). Finalmente formula su solución al problema de la fundamentación para casos en los que están involucrados objetos naturales animados, como los organismos.

Como he dicho, en esta sección vamos a utilizar su caso del diamante pero tendremos que modificar el ejemplo porque Sutton lo presenta en términos del diamante estando co-ubicado con una masa de átomos de carbono, y nosotros discutiremos el ejemplo en términos de un diamante co-ubicado con un trozo de materia compuesta de átomos de carbono

Creo que, intuitivamente, un diamante está co-ubicado con un trozo de materia de átomos de carbono. A lo mejor también está co-ubicado con la masa de átomos de carbono que Sutton menciona. Pero si intuitivamente afirmamos que, por ejemplo, una estatua de arcilla está co-ubicada con un trozo de arcilla, entonces deberemos afirmar también que un diamante está co-ubicado con un trozo de materia del tipo adecuado (cf. Campdelacreu 2016). 
La propuesta de Sutton para el caso del diamante no implica la existencia de dos objetos co-ubicados intrínsecamente compuestos porque presenta el caso en términos del diamante estando solamente co-ubicado con la masa de átomos de carbono; y las masas están, según Sutton, compuestas extrínsecamente. Sin embargo, aunque ella esté en lo correcto respecto a las masas, si es correcto lo dicho en el texto respecto de los diamantes estando también co-ubicados con trozos compuestos de átomos de carbono, seguimos teniendo un caso de objetos co-ubicados intrínsecamente compuestos.

Este es, entonces, el caso en términos del cual presentaremos primero la solución al problema de la fundamentación para casos de objetos co-ubicados intrínsecamente compuestos: un diamante, DIAMANTE, co-ubicado con un trozo del material adecuado, compuesto de átomos de carbono, $\mathrm{TROZO}^{\mathrm{C}}$-para que la explicación sea más ágil, de ahora en adelante llamaremos a este tipo de trozos de materia 'trozos de materiac'.

Veamos pues qué hecho fundamenta el hecho de que DIAMANTE y TROZO ${ }^{\mathrm{C}}$ tengan propiedades clasificatorias diferentes.

\subsubsection{Configuración interna mínima}

Los únicos objetos que tendremos en cuenta para el resto de la sección 3 serán los objetos intrínsecamente compuestos.

Para responder a la cuestión de la fundamentación necesitamos presentar lo que vamos a llamar 'configuración interna mínima' del componente material de un proceso ${ }^{\mathrm{e}}$. Se trata de ciertas relaciones específicas, que ahora presentaremos en detalle, que los componentes del componente material de los procesos ${ }^{\mathrm{e}}$ mantienen entre sí.

Primero, para el caso de DIAMANTE, y, de hecho, para todos los diamantes. La configuración interna mínima del componente material de un proceso ${ }^{\mathrm{e}}$ cuyo resultado es un objeto que tiene como propiedad clasificatoria la de ser un diamante es muy específica, se trata de la llamada 'red de diamante'. Así, análogamente a los casos anteriores, diremos que el hecho de que DIAMANTE tenga como propiedad clasificatoria la de ser un diamante está fundamentado en el hecho de que el proceso ${ }^{\mathrm{e}}$ del que es resultado tiene un componente material que ejemplifica la configuración interna mínima red de diamante.

Segundo, para el caso de $\mathrm{TROZO}^{\mathrm{C}}$, y para todos los casos de trozos de materia ${ }^{\mathrm{c}}$. La configuración interna mínima es disyuntiva; entre sus disyuntos podemos encontrar a la red de diamante pero también a la estructura propia del grafito, por ejemplo. Los componentes del componente material de un proceso $^{\mathrm{e}}$ ejemplifican esta configuración interna mínima en virtud de ejemplificar uno u otro de estos disyuntos.

Aquí es importante apuntar que, por ejemplo en este caso de los trozos de materiac,

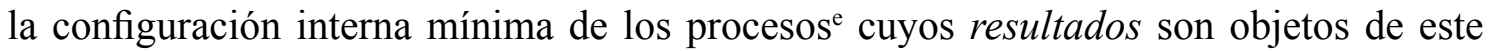
tipo, es, como pretende sugerir el nombre, la mínima configuración interna compartida 
por todos ellos. Es por esto que aunque, en un caso determinado, el objeto resultante pueda ejemplificar durante toda su existencia el disyunto que ejemplifica el componente material del proceso ${ }^{\mathrm{e}}$ del cual es resultado, esto no quiere decir que la configuración interna mínima del proceso $^{\mathrm{e}}$ del cual es resultado sea ese disyunto (por ejemplo la red de diamante). Al contrario, como hemos dicho, será la propiedad disyuntiva, que es la única común a todos los procesos ${ }^{\mathrm{e}}$ que tienen como resultado un objeto con la propiedad de ser un trozo de materia ${ }^{c}$ y que el componente material ejemplificará en virtud de ejemplificar el disyunto en cuestión. Una posible duda entorno a esta propuesta es que, si aceptamos que las configuraciones internas mínimas sean disyuntivas como las vistas, esto implique que cualquier disyunción de relaciones satisfecha por las partes de un componente material dé lugar a una nueva configuración interna mínima y con ello a un nuevo proceso ${ }^{\mathrm{e}} \mathrm{y}$, con ello, a un nuevo objeto resultante, co-ubicado con muchos otros. Esto puede llevar a una proliferación indeseada de objetos, muchos de ellos co-ubicados. Aquí solo dispongo de espacio para señalar lo siguiente. Creo que debemos diferenciar dos cuestiones/problemas diferentes: por un lado, la cuestión de qué determina que ciertos objetos existan. La otra cuestión es la que estamos tratando en este artículo: presuponiendo que los objetos existentes son los determinados por una respuesta correcta a la otra cuestión, ¿qué determina que dos objetos co-ubicados tengan diferentes propiedades clasificatorias? Es decir, al tratar el problema de la fundamentación ya presuponemos, legítimamente, que la solución al primer problema nos aportará una razón para determinar qué objetos existen y cuáles están co-ubicados. Así, al apelar a configuraciones internas mínimas disyuntivas, nos restringimos a aquellas disyunciones de relaciones entre partes de componentes materiales cuyos procesos ${ }^{\mathrm{e}}$ tienen como resultado objetos que existen según la respuesta correcta a la primera pregunta.

Así, análogamente a todos los casos vistos hasta ahora, diremos que el hecho que fundamenta el hecho de que $\mathrm{TROZO}^{\mathrm{C}}$ tenga como propiedad clasificatoria ser un trozo de materia $^{c}$ es el hecho de que el proceso ${ }^{\mathrm{e}}$ del cual es resultado tiene como componentes el

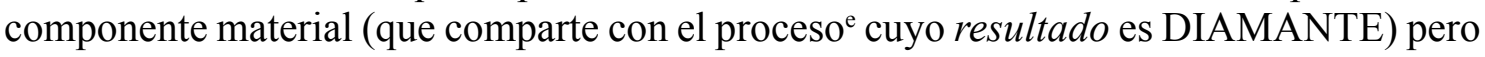
también la configuración interna mínima disyuntiva que acabamos de explicar, común a todos los $\operatorname{procesos}^{\mathrm{e}}$ que tienen como resultado a un trozo de materia ${ }^{\mathrm{c}}$.

El siguiente comentario debería ayudar a hacer más clara la propuesta. Los procesos ${ }^{\mathrm{e}}$ cuyos resultados son DIAMANTE y TROZO ${ }^{\mathrm{C}}$ comparten el mismo componente material (como era el caso para ESTATUA y ARCILLA). Sin embargo, difieren en otra característica, sus configuraciones internas mínimas, que siendo diferentes (una es disyuntiva y la otra no) son, sin embargo, compatibles dado que uno de los disyuntos de la configuración interna mínima disyuntiva es justamente la configuración interna mínima no disyuntiva. Es de esta manera que un mismo componente material puede ejemplificar las dos a la vez.

Así, la respuesta al problema de la fundamentación para el caso de DIAMANTE y $\mathrm{TROZO}^{\mathrm{C}}$ es perfectamente análoga a la del caso de ESTATUA y ARCILLA. El hecho que 
fundamenta el hecho de que DIAMANTE y $\mathrm{TROZO}^{\mathrm{C}}$ tengan diferentes propiedades clasificatorias es el hecho de que los procesos ${ }^{\mathrm{e}}$ de los que son resultados difieren en sus componentes, pues tienen distintas (aunque compatibles) configuraciones internas mínimas.

(Recuérdese aquí lo que dijimos para el caso de ARCILLA y ESTATUA en el apartado 3.1 sobre la apelación a hechos metafísicamente básicos, primitivos).

\subsubsection{Objetos co-ubicados intrínsecamente compuestos}

Esta solución al problema de la fundamentación es válida para todos aquellos casos en que dos objetos co-ubicados intrínsecamente compuestos son el resultado de procesos ${ }^{\mathrm{e}}$ que tienen diferentes (aunque compatibles) configuraciones internas mínimas. ¿Podemos afirmar que esto es lo que pasa en todos los casos de objetos co-ubicados intrínsecamente compuestos? Aquí vamos a ofrecer algunas razones para pensar que sí.

Este es el caso, por ejemplo, de una gran variedad de objetos del tipo de los minerales que son el resultado de procesos ${ }^{\mathrm{e}}$ cuyas configuraciones internas mínimas son estructuras (cristalinas) muy precisas. Estos objetos están co-ubicados con trozos de materia adecuados que son resultado de procesos ${ }^{\mathrm{e}}$ con estructuras internas mínimas más disyuntivas, aunque compatibles con las de los procesos ${ }^{\mathrm{e}}$ cuyos resultados son los objetos del tipo de los minerales. Este tipo de caso es perfectamente análogo al caso de DIAMANTE y TROZOC $^{\mathrm{C}}$.

Otra serie de casos que están sujetos al mismo análisis son los casos análogos a los siguientes dos casos: una montaña de hielo co-ubicada con un trozo de hielo (muy grande, claro) o un cerro co-ubicado con un trozo de marga. La configuración interna mínima de los procesos ${ }^{\mathrm{e}}$ cuyos resultados son las montañas de hielo (cerros de marga) requiere que las partes del componente material ejemplifiquen la forma de una montaña (cerro). La configuración puede ser disyuntiva pero todos los disyuntos comparten este requisito (y varían en otros). Sin embargo, la configuración interna mínima de los procesos ${ }^{\mathrm{e}}$ cuyos resultados son los trozos de hielo (marga) es más flexible en este aspecto y no todos sus disyuntos requieren que las partes de los componentes materiales ejemplifiquen una forma de montaña (cerro). Así, como las dos configuraciones internas mínimas incluyen diferentes disyuntos, son diferentes. Sin embargo, son compatibles porque comparten, al menos, alguno de sus disyuntos. Si esto es así, ya sabemos cómo solventar el problema de la fundamentación: el hecho que fundamenta el hecho de que una montaña de hielo (un cerro de marga) y el trozo de hielo (el trozo de marga) co-ubicado con ella tengan diferentes propiedades clasificatorias es el hecho de que los procesos ${ }^{\mathrm{e}}$ de los cuales son resultados difieren en características relevantes: sus diferentes (compatibles) configuraciones internas mínimas ejemplificadas por su componente material compartido.

Una serie final de casos a los cuales también podemos aplicar la solución propuesta: se trata de objetos intrínsecamente compuestos co-ubicados con agregados, también intrín- 
secamente compuestos (aquí asumiré que los agregados existen y están intrínsecamente compuestos). Por ejemplo, una montaña co-ubicada con un agregado de objetos más pequeños como rocas, hierba, etc., que sería el caso normal para las montañas, más que el caso considerado antes, aunque este también fuera posible. $\mathrm{O}$ un volcán y el agregado de objetos más pequeños co-ubicado con él, o una nube y el agregado de gotas de agua co-ubicado con ella, o un cubito de hielo y el agregado de moléculas de $\mathrm{H}_{2} \mathrm{O}$ co-ubicado con él. Los agregados y las masas parecen ser dos tipos diferentes de entidades, dado que, por ejemplo, los agregados parecen poder serlo de objetos muy variados entre sí, como en el caso de la montaña, pero no parece ser así para las masas. También parece diferenciarlos (acorde con la posición sobre sus condiciones de existencia más aceptada actualmente) el grado de dispersión tolerado por sus componentes, mucho más extremo en el caso de los agregados. Igualmente, los agregados y los trozos de materia parecen ser dos tipos de entidades diferentes, pues el grado de dispersión tolerado por los componentes de los agregados, como acabamos de decir, es considerado (por las posiciones más aceptadas actualmente) como siendo mucho mayor que el tolerado por los componentes de los trozos de materia. De aquí que otra cuestión interesante que (por cuestiones de espacio) tendré que dejar aquí meramente apuntada es si las masas y los trozos de materia son el mismo tipo de objeto. En cualquier caso, también cuáles sean exactamente las condiciones de existencia de todos ellos debería ser objeto de extensa argumentación. Aquí solo he podido apuntar las asunciones realizadas en el contexto de la discusión principal del artículo sobre el problema de la fundamentación.

La configuración interna mínima de los procesos ${ }^{\mathrm{e}}$ cuyos resultados son agregados es extremadamente disyuntiva. Por ejemplo, tiene multitud de diferentes disyuntos que requieren que las partes del componente material muestren una gran variedad de grados de dispersión. Por otro lado, la configuración interna mínima de los procesos ${ }^{\mathrm{e}}$ cuyos resultados son los objetos con los cuales estos agregados están co-ubicados no es disyuntiva en este aspecto o, al menos, mucho menos disyuntiva que la de los procesos ${ }^{\mathrm{e}}$ de los agregados. Por ejemplo, no tienen ningún disyunto que requiera que los componentes del componente material estén muy dispersos. Si esto es correcto podemos aplicar la misma estrategia que antes valiéndonos de las diferentes, compatibles, configuraciones internas mínimas de los procesos ${ }^{\mathrm{e}}$ relevantes.

Ahora, aunque hemos ofrecido una serie de distintos tipos de casos más que una razón general, creo que es razonable concluir a partir de sus análisis que todos los casos de objetos co-ubicados intrínsecamente compuestos son relevantemente análogos a uno u otro de los tres tipos de casos presentados. A lo mejor un objeto, co-ubicado con un trozo de materia adecuado, no es un mineral del tipo visto antes pero la configuración interna mínima del proceso ${ }^{e}$ del cual es resultado requiere que las partes de su componente material muestren una configuración muy específica; contra lo que es el caso para la configuración interna mínima del trozo de materia adecuado, más disyuntiva. O a lo mejor un objeto no es una montaña de hielo, pero la configuración interna mínima del proceso ${ }^{\mathrm{e}}$ del cual es re- 
sultado requiere (en todos los disyuntos) que las partes del componente material ejemplifiquen una forma externa específica, contra lo que es el caso para alguno(s) de los disyuntos de la configuración interna mínima del trozo de materia con el cual está co-ubicado. $\mathrm{O}$ a lo mejor un objeto no es una nube o una piedra, pero la configuración interna mínima de su proceso $^{\mathrm{e}}$ es mucho menos tolerante a la dispersión de los componentes del componente material que la del proceso $^{\mathrm{e}}$ del agregado con el cual está co-ubicado. En todos estos casos, sin embargo, las configuraciones internas mínimas de los objetos co-ubicados comparten alguno(s) de sus disyuntos y así, aunque son diferentes, son compatibles.

\section{Objeciones y respuestas}

En esta sección vamos a formular y responder brevemente a varias posibles objeciones a la propuesta que acabamos de presentar. También vamos a comparar la propuesta formulada con las de Kit Fine y Noël Saenz.

\subsection{Objetos sin procesos ${ }^{\mathrm{e}}$}

Una posible objeción a la propuesta que acabamos de presentar podría ser la siguiente: imaginemos que el tiempo no tuviese un inicio y que, desde siempre, hubieran existido una estatua y un trozo de arcilla co-ubicados. En un caso así tendríamos a dos objetos co-ubicados, con propiedades clasificatorias diferentes, pero sin posibilidad de explicarlo en base a sus diferentes procesos para empezar a existir (pues no tienen).

Veamos a continuación como podríamos responder a esta objeción. Primero, antes de analizar la posibilidad de la situación descrita en el párrafo anterior, creo que es importante puntualizar lo siguiente. La solución que estoy esbozando aquí al problema de la fundamentación hace un uso crucial del hecho de que los objetos son, necesariamente, resultado de procesos ${ }^{\mathrm{e}}$. Sin embargo, que los objetos lo sean es una tesis independiente, previa, a su uso en relación al problema de la fundamentación, en el sentido de que hay argumentos a favor de la existencia de tales procesos que son independientes de su uso en la solución propuesta al problema de la fundamentación; en este sentido, hemos visto en secciones previas que es intuitivo pensar que existen procesos ${ }^{\mathrm{e}}$, al menos en casos corrientes, y veremos a continuación otra razón para pensar que les son esenciales (al menos) a los objetos en cuestión aquí.

Así, retomando la cuestión planteada en el primer párrafo de la sección, analicemos cuidadosamente las asunciones involucradas. Primero, puede no haber un primer instante de tiempo. Segundo, los objetos materiales pueden existir sin ser el resultado de un proceso $^{\text {e; }}$ su naturaleza debe permitirlo.

Para responder a esta posible objeción planteada en el primer párrafo, no voy a discutir la primera asunción. Sí la segunda. Veamos que es más problemática de lo que pueda

Revista de Humanidades de Valparaíso, 2020, No 16, 61-87

(c) (1) @ $\odot$ CC BY-NC-ND 
parecer a primera vista. Si la naturaleza de los objetos materiales en cuestión aquí es tal que pueden existir sin ser el resultado de un proceso ${ }^{\mathrm{e}}$, entonces debe ser posible que, en un escenario donde el tiempo sí tuvo un inicio, un objeto material, como por ejemplo un tigre, empiece a existir de esta manera, es decir, se materialice instantáneamente de la nada. Parece claro que si un tigre puede no ser el resultado de un proceso ${ }^{\mathrm{e}}$ cuando el tiempo no tiene un inicio debería poder no serlo en un mundo posible en que el tiempo sí tiene un primer instante. Pues que un objeto pueda existir sin ser el resultado de un proceso de este tipo depende de que su naturaleza lo permita, no de que el tiempo, algo externo a él, lo permita.

Ahora, creo que esta posibilidad es altamente anti-intuitiva para los objetos con los cuales estamos lidiando. ¿Es realmente concebible que un tigre, un árbol, una catedral, una silla, un diamante, etc., se materialicen instantáneamente de la nada? Para estos casos creo que la respuesta intuitiva es negativa. Una posible complicación sería la siguiente: pensemos, por ejemplo, en nuestro tigre. ¿Qué pasaría si pudiera viajar en el tiempo? No son pocos los filósofos que defienden que este tipo de escenarios son concebibles, e incluso, metafísicamente posibles. Ahora bien, en la región espaciotemporal en la que aparecería el tigre después del viaje, este no existiría como resultado de un proceso $^{\mathrm{e}}$, sino que, más bien, se materializaría instantáneamente de la nada. Pero esto parece ir en contra de la propuesta que estamos esbozando. Veamos, sin embargo, que no es así: lo que la propuesta que estamos esbozando afirma es que el tigre no puede sino empezar a existir por primera (y única) vez como resultado de un proceso ${ }^{\mathrm{e}}$ pero es neutra sobre qué ha de pasar para que siga existiendo. Y en el caso del viaje en el tiempo el tigre no empieza a existir después de este sino que ya existía, y sigue existiendo después del viaje. Nuestra teoría es neutra sobre cómo los objetos persisten, que es lo relevante en este caso.

Sin embargo, aunque no sea concebible que un tigre, un árbol, una catedral, una silla, un diamante, etc., se materialicen instantáneamente de la nada, a lo mejor sí lo es para otros objetos... ¿a lo mejor para los simples? Pero, ¿cómo puede afectar esta posibilidad a la solución propuesta? Veámoslo.

Consideremos este caso: supongamos que el tiempo sí tiene un primer instante y que en este primer instante existe una multitud de simples (vamos a suponer aquí, por mor del argumento, que existen), entendidos como aquellos objetos materiales que no están compuestos por otros objetos materiales, esto es, son los objetos materiales a partir de los cuales (junto con otros factores) obtenemos el resto. Para ellos no tenemos, creo, intuiciones claras sobre si pueden existir o no sin ser el resultado de un proceso ${ }^{\mathrm{e}}$. Asumamos en pro de la discusión que pueden existir sin serlo. Además, supongamos que, en el primer momento de tiempo existente, están dispuestos de tal manera (pues de alguna deben estarlo) que componen un trozo de materia. Este trozo de materia, sin embargo, no ha tenido ningún proceso ${ }^{\mathrm{e}}$. 
Analicemos un poco más este caso: ya he dicho que no creo que tengamos intuiciones claras sobre si los simples, descritos del modo como hemos hecho, son realmente posibles. Indaguemos, sin embargo, sobre qué pasaría si realmente lo fueran. Creo que lo que este caso mostraría es que para cierto tipo de objetos muy determinados, los trozos de materia obtenidos a partir de simples que están en ciertas relaciones que no involucran, para su establecimiento, el paso del tiempo (si no fuera así ya podríamos hablar de un caso como los vistos hasta ahora), más que de un proceso ${ }^{\mathrm{e}}$ deberíamos hablar de un 'proceso contemporáneo para existir'. Se trataría de procesos con los mismos tipos de componentes que los procesos ${ }^{\mathrm{e}}$ pero tales que sus objetos resultantes no existirían después del proceso, sino a la vez, contemporáneamente, al proceso. Parece que lo más plausible sería pensar que se trataría de casos en que el objeto resultante tendría una composición intrínseca (entendida ahora en términos de procesos contemporáneos para existir, a los cuales podríamos llamar procesos ${ }^{\mathrm{c}}$ en lugar de procesos ${ }^{\mathrm{e}}$ ), o al menos este parecería ser el caso de nuestro trozo de materia ${ }^{3}$.

¿Qué consecuencias tendría el hecho de que objetos relevantes en nuestra discusión, como los trozos de materia mencionados, pudiesen ser resultados de procesos ${ }^{\mathrm{c}}$ en lugar de resultados de procesos ${ }^{\mathrm{e}}$ ? Primero, me gustaría volver a enfatizar que el que los objetos pudiesen ser resultado de dos tipos de procesos sería una tesis previa a su uso en la solución al problema de la fundamentación. Segundo, la consecuencia de lo que acabamos de ver sería que nuestra solución al problema de la fundamentación debería adaptar este resultado y pasaría a ser, también, disyuntiva. Así, deberíamos afirmar que los objetos relevantes para el problema de la fundamentación serían el resultado de $\operatorname{procesos}^{\mathrm{e}} \mathrm{o}$ de $\operatorname{procesos}^{\mathrm{c}}$.

Pero, ¿podría haber casos de objetos co-ubicados, ambos resultado de procesos ${ }^{\mathrm{c}}$ ? ¿En qué consistiría en esos casos la solución al problema de la fundamentación? Por ejemplo, no parece ininteligible el siguiente escenario: co-ubicado con nuestro trozo de materia podría haber un agregado de los simples, resultado también de un proceso $^{c}$, tal que la propiedad categórica del trozo de materia y del agregado no serían la misma.

Ya hemos dicho que los procesos ${ }^{\mathrm{c}}$ tendrían los mismos tipos de componentes que los procesos ${ }^{\mathrm{e}}$. Siendo esto así, para el caso que nos ocupa aquí la solución al problema de la fundamentación sería análoga a las vistas en la sección 3.2.2: el hecho que fundamenta el hecho de que tengan diferentes propiedades categóricas es el hecho de que los dos objetos son resultado de procesos ${ }^{\mathrm{c}}$ con configuraciones internas mínimas diferentes, aunque compatibles: como hemos visto las configuraciones internas mínimas de los agregados son mucho más disyuntivas que las de los trozos de materia.

\footnotetext{
${ }^{3}$ Claramente, es necesario precisar mucho más como sería posible la existencia de estos simples, así como cuál es exactamente la naturaleza de los procesos $^{c}$. Aquí, sin embargo, por razones de espacio, deberemos dejar la cuestión meramente apuntada.
} 
En definitiva, la posibilidad de estos casos tan particulares no sería, pues, un problema insalvable para nuestra propuesta, aunque la haría disyuntiva.

\subsection{Kit Fine, formas y procesos ${ }^{\mathrm{e}}$}

En esta sección vamos a presentar la solución de Kit Fine al problema de la fundamentación y la compararemos con la solución propuesta más arriba. Ello ayudará a perfilarla. De hecho, Fine nos servirá de ejemplo de teorías que, de un modo u otro, postulan la existencia de formas, y que las utilizan para solventar el problema de la fundamentación. Lo que digamos para las formas fineanas sería aplicable de forma análoga a estas otras teorías.

Kit Fine (cf. 1999; 2008) propone una teoría hilemórfica para los objetos materiales según la cual estos presentan a la vez un aspecto material y uno formal. La teoría de Fine consta de dos partes, la 'theory of rigid embodiment' que podríamos traducir como 'la teoría de la materialización rígida' para la relación de ser parte de atemporal y la 'theory of variable embodiment' que podríamos traducir como 'la teoría de la materialización variable' para la relación de ser parte de temporal.

Veamos primero, de manera muy resumida, suficiente para nuestros propósitos presentes, la teoría de la materialización rígida.Dados ciertos objetos $\mathrm{a}, \mathrm{b}, \mathrm{c}, \ldots \mathrm{y}$ dada cierta relación $\mathrm{R}$ en que estos objetos pueden o no estar en un determinado momento de tiempo, existe la operación de materialización rígida cuyo resultado es un nuevo objeto compuesto por los objetos $\mathrm{a}, \mathrm{b}, \mathrm{c}$... y la relación $\mathrm{R}$ (el principio de materialización rígida). La relación $\mathrm{R}$ sirve para modificar a los objetos componentes y el resultado es un todo, cuyos componentes están unidos por esta relación. Un objeto de este tipo es una materialización rígida, pues la forma $\mathrm{R}$ está materializada en una materia que no cambia. Un ejemplo: una esfera de metal (supongamos que nunca cambia de partes) es el resultado de aplicar este método de composición al metal y a la forma esfera. A su vez, el trozo de metal co-ubicado con esta es el resultado de aplicar este método de composición al metal y a la forma trozo.

Veamos ahora la teoría de la materialización variable. Dada una función o principio $\mathrm{F}$ adecuado que a instantes temporales les asigna entidades, existe la operación de materialización variable '/ /' cuyo resultado /F/ lo obtenemos a partir del principio F (el 'principio de la materialización variable'). Los varios objetos que el principio selecciona en diferentes momentos de tiempo son las manifestaciones de la materialización variable $/ F /$. En contraste con el caso anterior, la materia de una materialización variable no está dada de forma independiente de la forma o principio, sino que está especificada por el principio mismo. Un ejemplo: un coche es una cierta materialización variable, $/ \mathrm{C} /$, donde el principio $\mathrm{C}$ selecciona en cada momento de tiempo en que el coche existe una cierta materialización rígida: las partes más relevantes del coche en la configuración característica 
de los coches. Otro ejemplo, el caso, ahora más realista, de una esfera de metal. En este caso tenemos el principio de materialización variable que selecciona en cada momento de su existencia una materialización rígida del tipo visto antes, pero en cada instante la materialización rígida seleccionada puede ser un poco diferente. Y análogamente para el caso más realista del trozo de metal co-ubicado con la esfera.

La teoría de las materializaciones rígidas/variables le permite a Fine dar la siguiente respuesta al problema de la fundamentación, aunque cabe señalar que el ámbito de aplicación de la misma va más allá de este uso. En el caso de la esfera de metal y el trozo de metal, tanto en los casos de materializaciones rígidas como variables, los objetos co-ubicados tienen diferentes formas como parte: el principio de materialización rígida y el principio de materialización variable.. Para el caso de las materializaciones rígidas, la misma teoría lo especifica (véase los artículos citados al principio de la sección). Para el caso de las materializaciones variables, Fine mismo lo sugiere $(1999,73)$, y según una comunicación personal, Koslicki afirma que lo acepta (2008, 78-79, n. 5). Esta diferencia explicaría la diferencia en sus propiedades clasificatorias.

Ahora, creo que un punto a favor de la teoría que hemos elaborado nosotros más arriba es el siguiente. En la subsección anterior hemos intentado defender que los objetos con los que estamos lidiando aquí (los involucrados en el problema de la fundamentación) tienen como propiedad esencial, independientemente del problema de la fundamentación, el que sean resultado de un proceso adecuado. Nosotros hemos propuesto entender tales procesos en términos de procesos $^{e}$ (dejamos a un lado el caso de los simples/trozo de materia; su posible existencia no cambia lo que argumentaremos a continuación, dado el predominio de los casos en que es esencial al objeto ser resultado de un proceso ${ }^{\mathrm{e}}$ del tipo adecuado). Pues bien, si esto es así, las formas fineanas de los objetos se vuelven redundantes para solucionar el problema de la fundamentación. El hecho de que dos objetos co-ubicados tengan propiedades clasificatorias diferentes ya queda previamente fundamentado en el hecho de que los dos procesos ${ }^{\mathrm{e}}$ de los que son resultado son relevantemente diferentes. Claro está, nuestro oponente podría argumentar que aunque ciertos procesos para empezar a existir sean esenciales a los objetos en cuestión, no deben entenderse de la manera propuesta, de manera que no nos servirían para solucionar el problema de la fundamentación. Claro está, también, que para que podamos considerar esta objeción, nuestro oponente debería indicarnos cómo deberíamos entender estos procesos de forma alternativa y por qué su propuesta sería mejor que la nuestra. Aquí deberemos dejar el debate en este punto.

En cualquier caso, aquí es necesario señalar que, para poder decidir finalmente entre una de las dos teorías deberíamos analizar primero cada uno de los usos que puedan tener y sus interacciones. En este artículo no podré sino restringirme a la comparación parcial que acabamos de esbozar, centrada exclusivamente en la aplicación de ambas teorías al problema de la fundamentación. 
Antes de pasar a comparar la propuesta que estamos esbozando con la teoría de Noël Saenz, creo que puede ser interesante la siguiente observación relacionada con el hecho de que la ventaja que acabamos de argumentar que nuestra teoría presenta respecto a la de Fine depende, claro, del hecho de que la nuestra se basa en la existencia de procesos ${ }^{\mathrm{e}}$, sobre los cuales podríamos afirmar lo siguiente: antes teníamos dos objetos coincidentes;

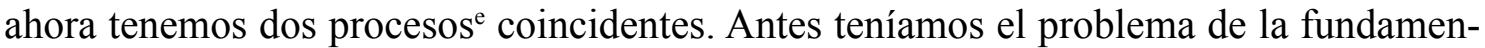
tación para los objetos, ahora, simplemente, tenemos el problema de la fundamentación para los procesos, que son de tipos diferentes.

Aunque durante todo el artículo hayamos asumido que los procesos ${ }^{\mathrm{e}}$ de los cuales ESTATUA y ARCILLA (por ejemplo) son resultado son diferentes, creo que, de hecho, todo lo que hemos dicho es compatible con que estos procesos no sean dos sino uno. En el siguiente sentido: ESTATUA y ARCILLA serían dos resultados diferentes de uno y el mismo proceso, en virtud de aspectos diferentes de este. Esto sería así en el mismo sentido en que, por ejemplo, el proceso de cortar un árbol puede tener dos resultados diferentes en virtud de diferentes aspectos del mismo: que el árbol muera y que el leñador resulte herido.

\subsection{Noël Saenz, estatuas y artefactos}

Para finalizar me gustaría analizar brevemente la propuesta de Saenz (cf. 2015), para responder al problema de la fundamentación. En concreto, me gustaría centrarme en su propuesta para el caso de las estatuas y otros artefactos y compararla con la que hemos desarrollado nosotros antes. Primero debemos mencionar que según Saenz la relación de fundamentación se da entre todo tipo de entidades. Aunque ya hemos señalado antes que no es de esta manera como la entendemos aquí, en esta sección vamos a reproducir el uso del autor; por otra parte, ya mencionamos más arriba que Saenz (cf. 2015) cree razonable pensar que existen conexiones sistemáticas entre diferentes maneras de entender los relata de la relación. También debemos apuntar que, según Saenz, las propiedades clasificatorias de los objetos no están, en ningún caso, o al menos, en ninguno de los que menciona en el artículo antes citado y a los que aludiremos en esta sección, ni parcialmente fundamentadas en relaciones que las partes de los objetos puedan tener con entidades diferentes a ellas mismas.

Según Saenz lo que genera el problema de la fundamentación, por ejemplo en el caso de una estatua y el trozo de arcilla co-ubicado con ella, es que estos tienen las mismas partes configuradas de la misma manera. Pero entonces, ¿qué explica la diferencia en sus propiedades clasificatorias?

Saenz usa el siguiente ejemplo. Pyra es una estatua en forma de pirámide compuesta por tres bloques: A, B, y C (en orden descendiente). Co-ubicado con Pyra está Lumpy, un trozo de arcilla. 
La propuesta de Saenz es la siguiente. Lo que fundamenta Pyra o Lumpy son ciertas configuraciones de sus partes -donde las configuraciones de objetos son hechos que tienen objetos y relaciones entre ellos como constituyentes. Y la configuración que es el fundamento de Lumpy no es la misma que la que fundamenta Pyra. El fundamento de Lumpy consta de los siguientes dos hechos: el hecho de que $A$ está en contacto con $B \mathrm{y}$ el de que $B$ está en contacto con $C$. El fundamento de Pyra consta de los siguientes dos hechos: el hecho de que $A$ está en Kontacto con $B$ y el de que $B$ está en Kontacto con $C$, donde $x$ está en Kontacto con $y$ si y solamente si la base más ancha de $x$ está en (más 0 menos) contacto completo con la base más estrecha de $y$.

El punto clave de la solución de Saenz es que lo relevante para fundamentar la propiedad clasificatoria de un objeto no es meramente qué partes tiene y como estas partes están configuradas, pues Pyra y Lumpy comparten todo esto. Lo que es relevante para fundamentar qué es un objeto es, muchas veces, algunas y solo algunas, configuraciones de sus partes. Es precisamente por esto que podemos tener dos objetos con las mismas partes organizadas de la misma manera y que, sin embargo, tengan propiedades clasificatorias diferentes.

Lo que me gustaría mostrar brevemente en lo que sigue es que, al contrario de lo que defiende Saenz, y tal y como es el caso en la propuesta que hemos defendido antes, debemos apelar en al menos algunos casos, a relaciones que las partes de un objeto mantienen con entidades externas a ellas para poder proporcionar una respuesta completa al problema de la fundamentación.

Para Saenz no es esencial a una escultura, ni a ningún artefacto, que sea creado por un ser humano, aunque puede estarlo, claro. Los fundamentos de sus propiedades clasificatorias son, como hemos visto, sus partes y algunas (o todas, en algunos casos) de las relaciones que estas mantienen entre sí. Creo que si esto es así, la propuesta de Saenz no puede proporcionar una respuesta satisfactoria al siguiente tipo de casos.

Un caso como el siguiente parece ser claramente posible: imaginemos una escultura formada por una serie de barras rectas dispuestas verticalmente y otro par de barras que, dispuestas de forma aproximadamente horizontal, cruzan las anteriores formando una serie de ángulos concretos con las primeras. Supongamos además que se trata de una escultura para la cual no importa el material de qué estén hechas estas barras. Solo su número, cuan largas son y la manera exacta en qué están dispuestas entre sí formando los ángulos concretos que forman. Cualquier variación en esto implica que la escultura deja de existir. Por otro lado, parece igual de posible imaginar que co-ubicado con ella de forma permanente existe un artefacto para representar de cierta manera material muy específica las complejas relaciones entre una serie de números concretos. Podemos imaginar que dos personas trabajan en la creación de los dos objetos, cada una con un propósito diferente. Las dos lo consiguen y los dos objetos empiezan a existir al mismo tiempo. Recordemos que, con todo, para Saenz, para este tipo de objetos las diferentes relaciones 
en que puedan estar con objetos externos son irrelevantes a la hora de fundamentar sus propiedades clasificatorias, y que esto es compatible con que, en ciertos casos, estos objetos sean creados por personas. Ahora, imaginemos que para tal artefacto, al igual que en el caso de la escultura, no importa el material de qué estén hechas las barras, solo su número, cuan largas son y la manera exacta en qué están dispuestas entre sí formando los ángulos concretos que forman. Cualquier variación en esto implica que el artefacto en cuestión deja de existir pues ya no es apto para llevar a cabo el tipo de representación material en cuestión. Para hacernos una idea de en qué podría consistir este tipo de representación material, imaginemos que el artefacto representa el número más grande de la serie en base al número de barras de que está hecho y que representa a los diferentes números de la serie en base al número de centímetros que miden las diferentes barras en cierto orden específico (de derecha a izquierda y luego de arriba abajo) y que representa a ciertas relaciones que mantienen estos números en base al número de grados que miden los ángulos formados por ciertas barras, etc. En definitiva, parece que con un poco de paciencia podemos imaginarnos una escultura y un artefacto representacional de cierto tipo muy determinado con exactamente las mismas configuraciones de sus partes como posibles fundamentos de sus propiedades clasificatorias. Pero si esto es así, para este tipo de caso la teoría de Saenz no nos puede ofrecer una respuesta adecuada al problema de la fundamentación. Sí, en cambio, puede hacerlo la teoría que hemos defendido más arriba: los procesos ${ }^{\mathrm{e}}$ de los cuales son resultado tienen como componentes diferentes relaciones con diferentes estados mentales (como mínimo), unos relacionados con las esculturas y los otros relacionados con los artefactos de representación.

\section{Conclusión}

En este artículo hemos presentado una nueva respuesta al problema de la fundamentación, uno de los más severos con que se enfrentan los defensores del co-ubicacionismo. Para ello hemos integrado algunas de las tesis de la propuesta de Sutton, que hemos visto que, sin embargo, es incompleta, y así inadecuada para sus propósitos. También hemos argumentado, brevemente, que esta estrategia presenta ciertas ventajas respecto de otras rivales, como la de Fine y la de Saenz. Como hemos argumentado, el co-ubicacionismo puede responder al que puede ser su mayor reto teniendo en cuenta que los objetos materiales del sentido común científicamente refinado son resultado de procesos ${ }^{\mathrm{e}}$. 


\section{Agradecimientos}

La investigación necesaria para este artículo ha sido financiada por el proyecto del Gobierno de España FFI2016-80588-R

\section{Referencias bibliográficas}

Bliss, R., Trogdon, K. (2014). Metaphysical Grounding. The Stanford Encyclopedia of Philosophy. https://plato.stanford.edu/archives/win2016/entries/grounding/. Acceso: 10/11/2020.

Campdelacreu, M. (2015). Objetos Materiales, Partes y Tiempo. En J.L. Prades (ed.), Cuestiones de Metafisica, pp. 255-290. Madrid: Tecnos. https://www.tecnos.es/ficha.php?id=4208018

Campdelacreu, M. (2016). Sutton's Solution to the Grounding Problem and Intrinsically Composed Colocated Objects. Crítica, 48, 77-92. https://doi.org/10.22201/iifs.18704905e.2016.223

Correia, F., Schnieder, B. (eds.) (2012). Metaphysical Grounding: Understanding the Structure of Reality. Cambridge: Cambridge University Press. https://doi.org/10.1017/ CBO9781139149136

deRosset, L. (2013). Grounding Explanation. Philosophers'Imprint, 13, 1-26. http://hdl.handle. net/2027/spo.3521354.0013.007

Fine, K. (1999). Things and Their Parts. Midwest Studies 23, 61-74. https://doi.org/10.1111/14754975.00004

Fine, K. (2003). The Non-Identity of a Material Thing and Its Matter. Mind, 112, 195-234. https:// doi.org/10.1093/mind/112.446.195

Fine, K. (2008). I-Kit Fine: Coincidence and Form. Aristotelian Society Supplementary Volume, 82, 101-118. https://doi.org/10.1111/j.1467-8349.2008.00164.x

Gibbard, A. (1975). Contingent Identity. Journal of Philosophical Logic, 4, 187-221. https://doi. org/10.1007/BF00693273

Koslicki, K. (2008). The Structure of Objects. Oxford: Oxford University Press. https://doi. org/10.1093/acprof:oso/9780199539895.001.0001

Raven, M. (2015). Ground. Philosophy Compass, 10(5), 322-333. https://doi.org/10.1111/ phc3.12220

Saenz, N. B. (2015). A Grounding Solution to the Grounding Problem. Philosophical Studies, 172, 2193-2214. https://doi.org/10.1007/s11098-014-0405-x

Sutton, C.S. (2012). Colocated Objects, Tally-Ho: A Solution to the Grounding Problem. Mind, 121, 703-30. https://doi.org/10.1093/mind/fzs078

Trogdon, K. (2013). An Introduction to Grounding. En M. Hoeltje, B. Schnieder, A. Steinberg (eds.), Varieties of Dependence, pp. 97-122. Germany: Philosophia Verlag.

Trogdon, K. (2018). Grounding-Mechanical Explanation. Philosophical Studies, 175, 1289-1309. https://doi.org/10.1007/s11098-017-0911-8 
Una nueva solución al problema de la fundamentación

Marta Campdelacreu

Wasserman, R. (2017). Material Constitution. The Stanford Encyclopedia of Philosophy. https:// plato.stanford.edu/archives/fall2017/entries/material-constitution/. Acceso: 15/05/2019.

Wilson, A. (2018). Metaphysical Causation. Noûs, 52, 723-51. https://doi.org/10.1111/nous.12190 
